\title{
Article \\ A Physically Meaningful Relativistic Description of the Spin State of an Electron
}

\author{
Yaakov Friedman (D)
}

check for updates

Citation: Friedman, Y. A Physically Meaningful Relativistic Description of the Spin State of an Electron. Symmetry 2021, 13, 1853. https:// doi.org/10.3390/sym13101853

Academic Editors: Sergei D. Odintsov and Palle E. T. Jorgensen

Received: 5 July 2021

Accepted: 10 August 2021

Published: 3 October 2021

Publisher's Note: MDPI stays neutral with regard to jurisdictional claims in published maps and institutional affiliations.

Copyright: (C) 2021 by the author. Licensee MDPI, Basel, Switzerland. This article is an open access article distributed under the terms and conditions of the Creative Commons Attribution (CC BY) license (https:/ / creativecommons.org/licenses/by/ $4.0 /)$.
Jerusalem College of Technology, Departments of Mathematics and Physics, Extended Relativity Research Center, P.O. Box 16031, Jerusalem 91160, Israel; friedman@jct.ac.il

\begin{abstract}
We introduced a new model to present the states of a two-state quantum system. The space is the complexified Minkowski space. The Lorentz group acts by the linear extension of its action on the four-vectors. We applied this model to represent the spin state of an electron or any relativistic spin $1 / 2$ particle. The spin state of such particle is of the form $U+i S$, where $U$ is the four-velocity of the particle in the lab frame, and $S$ is the $4 \mathrm{D}$ spin in this frame. Under this description, the transition probability between two pure spin states $\varrho_{1}$ and $\varrho_{2}$ of particles moving with the same velocity are defined by use of Minkowski dot product as $\frac{1}{2}<\varrho_{2} \mid \varrho_{1}>$. This transition probability is Lorentz invariant, coincide with the quantum mechanics prediction and thus agree with the experimental results testing quantum mechanics predictions based on Bell's inequality. For a a particle of mass $m$ and charge $q$ with the spin state $\varrho$, the total momentum is mc $\varrho$ and the electromagnetic momentum is $q \varrho$. This imply that the Landé $g$ factor for such particles must be $g=2$. We obtain an evolution equation of the spin state in an electromagnetic field which defines correctly the anomalous Zeeman effect and the fine structure splitting.
\end{abstract}

Keywords: spin state; relativistic spin; transition probability; Bell's inequality; spin triple product

\section{Introduction}

Two-level quantum mechanical systems, or two-state systems, are the simplest among quantum systems, yet they are enormously useful and have a wide range of applications. They can explain the most fundamental quantum phenomena, such as interference patterns. However, they are also used to model complicated processes, such as nuclear magnetic resonance and neutrino oscillation. In addition, the two-state formalism is the basis of quantum computing. An important example of a two-state quantum system is the spin state of a spin $1 / 2$ particle, such as an electron.

The usual definition of spin is an intrinsic form of angular momentum carried by elementary particles. It is claimed that spin has no classical analogue [1]. The existence of electron spin angular momentum has been inferred from the Stern-Gerlach experiment [2], in which silver atoms exposed to a non-uniform magnetic field displayed two possible discrete angular momenta. The existence of spin follows also from the anomalous Zeeman effect. The pure state space of a two-level quantum mechanical system are qubits in quantum computing. Many experiments prove the existence of both an electron's angular and magnetic momentum in various physical phenomena.

The relativistic quantum theory of electron spin was developed by Dirac [3] by representing the state with bi-spinors and using the spin 1/2 representation of the Lorentz group. However, there do exist alternative approaches to the relativization of spin, for example see $[4,5]$.

Electron spin can be treated partially by use of classical electrodynamics [6] and special relativity. Uhlenbeck and Goudsmit hypothesized that the electron's magnetic momentum $\mu$ and its angular momentum s are related by

$$
\boldsymbol{\mu}=\frac{g q}{2 m c} \mathbf{s}
$$


where $q$ and $m$ are, respectively, the charge and mass of the electron, $c$ is the speed of light in vacuum and $g$ is the Landé factor. The classical value for $g$ is 1 . They observed that the anomalous Zeeman effect could be explained if we assume $g=2$. However, under this assumption, the observed fine structure interval is one half of the one predicted. Thomas [7] has shown that if one uses relativistic kinematics to describe the precession of the frame comoving to the electron, then $g=2$ explains both the anomalous Zeeman effect and the observed fine structure interval. This is an indication that spin should be treated within relativity theory.

We propose here a new physically meaningful relativistic presentation of the state space of a two-state quantum system and the spin of spin $1 / 2$ particles. The measuring process defines certain geometric properties on the state space. It was shown in [8] that the state space of a two-state system satisfying certain physically significant properties is isometric to the dual (or pre-dual) of a complex spin factor. In the algebraic formulation of non-relativistic quantum mechanics, pure spin states are modeled by rank one projection operators. In [9], it was shown that relativization of the spin model requires replacing these projections with tripotent operators in a 4D spin domain in the Cartan classification [10].

The $4 \mathrm{D}$ spin domain is built on the Euclidean geometry of $\mathbb{R}^{4}$ or $\mathbb{C}^{4}$. The geometry of spacetime is the hyperbolic geometry of Minkowski spacetime. By replacing the Euclidean geometry in the 4D spin domain with Minkowski one leads to a complexified Minkowski space endowed with a triple product. We call it the Relativistic Two-State Space and denote it by $M_{\mathcal{c}}$. We obtain explicit description for pure states in $M_{c}$ and show that it can be used as a state space of two-state quantum systems. Explicit formulas for transition probabilities are obtained. The usual Lorentz group representation on Minkowski space is linearly extended to $M_{c}$ and the set of pure states is invariant under the action of the group. Thus, this state space representation is a relativistic one.

For example, $M_{c}$ can be used to represent the relativistic spin of an electron. The spin is represented by a vector under this representation and not by a bi-spinor, as in Dirac formulation. The transition probability between two pure spin states coincides with the quantum mechanics prediction. The Landé $g$ factor for such particles must be $g=2$. We obtain an evolution equation of the spin state in an electromagnetic field which defines correctly the anomalous Zeeman effect and the fine structure splitting.

\section{Relativistic State Space of a Two-State Quantum System}

In the algebraic formulation of non-relativistic quantum mechanics, pure states are represented by rank one projections on an abstract state space. A projection is a mathematical representation of a filtering process which accepts incoming particles and outputs particles with the state associated with the given filter. Mathematically, an operator $p$ is a projection if it satisfies the identity $p^{2}=p$. Two projections are orthogonal if the states corresponding to one of them do not pass the filtering process defined by the second one. We can define a partial ordering on the set of projections by defining the projection $p$ to be less than the projection $q$ if there is a projection $r$, orthogonal to $p$, such that $q=p+r$. The rank one projections are minimal in the projection ordering, expressing the fact that the corresponding state cannot be decomposed into a sum of two orthogonal states. A quantum system is called a two-state system if there are not more than two orthogonal projections on the state space. All projections belong to a cone defined by the identity operator $I$, meaning that $p<I$ and $p$ commutes with $I$. Since all such states are stationary, we may assign to the identity operator the meaning of an operator representing the time observable in the model. Since, in Relativity, time in one inertial system is not the time in another system moving with respect to the first one, the time operator defined by $I$ in the first system will not be the identity operator in the second one. Thus, the above algebraic model cannot become relativistic without weakening its assumptions.

Any projection $p$ is also a tripotent, meaning $p^{3}=p$. The set of tripotents is larger than the set of projections, and there are tripotents which are not projections. We may associate a filtering process to any tripotent, define orthogonality and a partial order on the 
set of tripotents [11]. Under this ordering, minimal projections become minimal tripotents. In order to be able to define tripotents, we do not need a binary algebraic operation. Rather, it is enough to have a ternary operation which gives meaning to the identity $p^{3}=p$. For example, the triple product defined by the geometry of bounded symmetric domains is such a ternary operation. The ball of bounded operators on a Hilbert space, occurring in the algebraic formulation of quantum mechanics, is a bounded symmetric domain. However, there are also other bounded symmetric domains. An important one is the spin domain in Cartan's classification. In [9], we have shown that in order that the state of the spin of spin 1/2 particles be Lorentz invariant, we need to use minimal tripotents to describe operators associated to pure states. However, in order to have the model invariant under the usual Lorentz group representation on Minkowski spacetime, we must modify the model, as follows.

We introduce the Relativistic Two-State Space to represent the state space of a two-state quantum system. As a space, it is a complexified Minkowski space $M_{\mathcal{C}}=M \oplus i M$. Its real part is the Minkowski space of four-vectors, such as the four-velocity and the fourmomentum, which are independent of the choice of orientation of the space. The imaginary part, on the other hand, may consist of four-pseudo-vectors, such as angular velocity and magnetic momentum, which change their direction with a change of orientation. We introduce a pseudo-scalar $i$ which also changes sign with a change of orientation. Thus, elements of $M_{c}$ are independent of the choice of the orientation. A similar idea is used in electromagnetism in the definition of the Faraday vector $\mathbf{F}=\mathbf{E}+i \mathbf{B}$.

Denote the natural basis of $M_{c}$ by $e_{0}, e_{1}, e_{2}, e_{3}$. Any element $x \in M_{c}$ can be written as $x=x^{\mu} e_{\mu}$, for $x^{\mu} \in \mathbb{C}$. The dot product of two elements $x, y \in M_{c}$ is defined as

$$
\langle y \mid x\rangle=\eta_{\mu \nu} y^{\mu} x^{\nu}, \quad \eta=\operatorname{diag}(1,-1,-1,-1) .
$$

Note that this dot product is linear in both terms and is complex valued. The norm of a vector $x \in M_{\mathcal{C}}$, which is an extension of the Minkowski norm, is defined as $x^{2}=\eta_{\mu \nu} x^{\mu} x^{v}=\langle x \mid x\rangle$. and its complex conjugation as $\bar{x}=\bar{x}^{\mu} e_{\mu}$.

The algebraic structure on $M_{c}$ is defined, similar to [11], by use of a triple product

$$
\{a, b, c\}=\langle b \mid a\rangle c+\langle b \mid c\rangle a-\langle\bar{a} \mid c\rangle \bar{b} .
$$

This is a modification of the triple product defined by a bounded symmetric domain of type IV in Cartan's classification. This product corresponds to the product of the vector part of quaternions. Such a product is derived in ([12], p. 91) to describe the magnetic force between currents using the symmetries of the problem. This triple product was used in [13] to solve the relativistic evolution of a charge in an electromagnetic field. Using a new dynamic variable and the above triple product make the evolution conformal and lead to analytic solutions.

An element $v \in M_{\mathcal{C}}$ is called a tripotent if $v=\{v, v, v\}$. Using (3) for a tripotent $v$, we have $v=2 v^{2} v-\langle\bar{v} \mid v\rangle \bar{v}$. Thus, if $\langle\bar{v} \mid v\rangle=0$ and $v^{2}=1 / 2, v$ is a tripotent. If we decompose such a tripotent $v$ into real and imaginary parts as $v=\frac{1}{2}(u+i w)$, then $\langle\bar{v} \mid v\rangle=0$ implies $u^{2}+w^{2}=0$, and $v^{2}=1 / 2$ implies $\langle u \mid w\rangle=0$ and $u^{2}-w^{2}=2$. From this, it follows that

$$
v=\frac{1}{2}(u+i w), \text { where } u^{2}=1, w^{2}=-1 \text { and }\langle u \mid w\rangle=0
$$

is a tripotent. Note that $\bar{v}$ is also a tripotent.

Two tripotents $v, u$ are orthogonal if $\{v, u, z\}=0$ for any $z \in M_{c}$. For example, if a tripotent $v$ is defined by (4), then, using (3), it follows that $\bar{v}$ is orthogonal to $v$. Moreover, $v+\bar{v}=u$. In addition, from (3), it follows that $u$ is a tripotent and, from the definition of partial order, we have $v<u$. Direct calculation shows that the only non-zero tripotents in $M_{c}$, in addition to the ones defined by (4), are real vectors $u$ satisfying $u^{2}=1$ and purely imaginary $i w$ satisfying $w^{2}=-1$. For the last two types of tripotents, there are no tripotents orthogonal to them, implying that they are maximal in the patrial ordering. A 
tripotent is minimal if it cannot be decomposed as a sum of mutually orthogonal tripotents. The tripotents $v$ defined by (4) are a minimal tripotents. Thus, in $M_{\mathcal{c}}$, there are no more than two orthogonal tripotents. Such a triple product space is said to be of rank two.

To be able to define the notion of transition probability, we need to define the pure state associated with a minimal tripotent. As in the algebraic formulation of quantum mechanics, the state associated with a minimal tripotent $v$ is a functional of norm one belonging to the dual space $M_{c}^{*}$ of $M_{c}$, with value 1 on $v$. The dot product (2) on $M_{c}$ allows one to identify each element $y \in M_{c}$ as a functional $\check{y}(x)=k\langle y \mid x\rangle$, where the constant $k$ depends on the normalization. The functional $\breve{v}$ associated to a minimal tripotent $v$ defined by (4) is

$$
\check{v}(x)=2\langle v \mid x\rangle=\langle 2 v \mid x\rangle .
$$

The constant 2 is needed in order to have $\breve{v}(v)=2\langle v \mid v\rangle=1$. The need of this 2 follows also from the fact that a minimal tripotent has norm one as an operator, but is not of norm one with respect to the Minkowski norm. Thus, the pure state corresponding to the tripotent $v=\hat{\varrho}$ from (4) should be

$$
\varrho=u+i w
$$

in order that $\langle\varrho \mid \hat{\varrho}\rangle=1$. Two states are called orthogonal if their corresponding tripotents are orthogonal. Since the triple product on $M_{\mathcal{C}}$ is of rank two, our model can be used to represent the state space of two-state quantum systems.

To make our model relativistic, we need to define a representation of the Lorentz group on $M_{c}$. For a relativistic model, a pure state in one inertial system must also be pure in another system. This means that any operator $\Lambda$ on $M_{c}$ from the representation of the group should transform a minimal tripotent, defined by (4), to a minimal tripotent. Obviously, if we define $\Lambda$ to be the complex-linear extension of the Lorentz transformation from Minkowski space $M$ to its complexification $M_{\mathcal{C}}$, it will possess the above property. Thus, $M_{\mathcal{C}}$ can be used to represent the relativistic state space of two-state quantum systems.

\section{Transition Probabilities of the Non-Relativistic Spin State of Spin 1/2 Particles}

From now on, we apply our model to describe the state of the spin of a spin $1 / 2$ particle. In this section, we treat first the non-relativistic case. The same treatment is valid for a relativistic particle in the comoving frame. As mentioned in ([14], p. 120), in non-relativistic quantum mechanics, a particle in a pure spin state has a definite direction of spin (more precisely, there exists a direction in which the spin component has definite value $1 / 2$ ). The spin state $\varrho$ of such a particle is defined by a space-like unit vector $\mathbf{a} \in \mathbf{R}^{3}$ representing the direction of the spin, with the meaning that the rotation is in the plane perpendicular to a clockwise or counter-clockwise, depending on the choice of orientation. This vector is called the Bloch vector [15], and the collection of all Bloch vectors is called the Bloch sphere. The Bloch sphere is a unit 2-sphere, representing the pure state space of a two-level quantum mechanical system, with antipodal points corresponding to a pair of mutually orthogonal state vectors. It is used in quantum mechanics and quantum computing.

Embed this vector a into $M_{c}$ by defining the four-vector $a=(0, \mathbf{a})$. In quantum mechanics, we associate with this state a spin state operator $\hat{\varrho}$, which is a rank one projection matrix onto this state. In our model, we replace this minimal projection by a minimal tripotent $v=\hat{\varrho}$ in $M_{c}$ defined by (4) and the corresponding pure state $\varrho$, defined by (6). Since the real part $u$ has the meaning of four-velocity, we may replace it with the vector $e_{0}$ representing the four-velocity of a rest particle in Minkowski space. Since the spin, representing the rotation of the particle, is a pseudo-vector, it belongs to the imaginary part of the spin state. Thus, the pure spin state operator $\hat{\varrho}$ and the corresponding pure state $\varrho$ are

$$
\hat{\varrho}=\frac{1}{2}\left(e_{0}+i a\right), \quad \varrho=e_{0}+i a
$$


In the matrix representation of the spin domain using Pauli matrices (see [15] p. 172 and [11] Section 3.3.6), this operator becomes the rank one projection used in non-relativistic quantum mechanics to represent the spin operator.

To determine the transition probability between two pure spin states $\varrho_{1}$ and $\varrho_{2}$ corresponding to minimal tripotents $v_{1}$ and $v_{2}$, respectively, let a denote the spin direction of $\varrho_{1}$, and let $\mathbf{b}$ denote the spin direction of $\varrho_{2}$. Since $|\mathbf{a}|=|\mathbf{b}|=1$, the usual dot product in $\mathbb{R}^{3}$ is $\mathbf{a} \cdot \mathbf{b}=\cos \theta$, where $\theta$ is the angle between the spin directions, see Figure 1 . Extend these $3 \mathrm{D}$ vectors to $4 \mathrm{D}$ vectors $a$ and $b$, respectively. Then, from (7), it follows that

$$
\varrho_{1}=e_{0}+i a, \varrho_{2}=e_{0}+i b .
$$

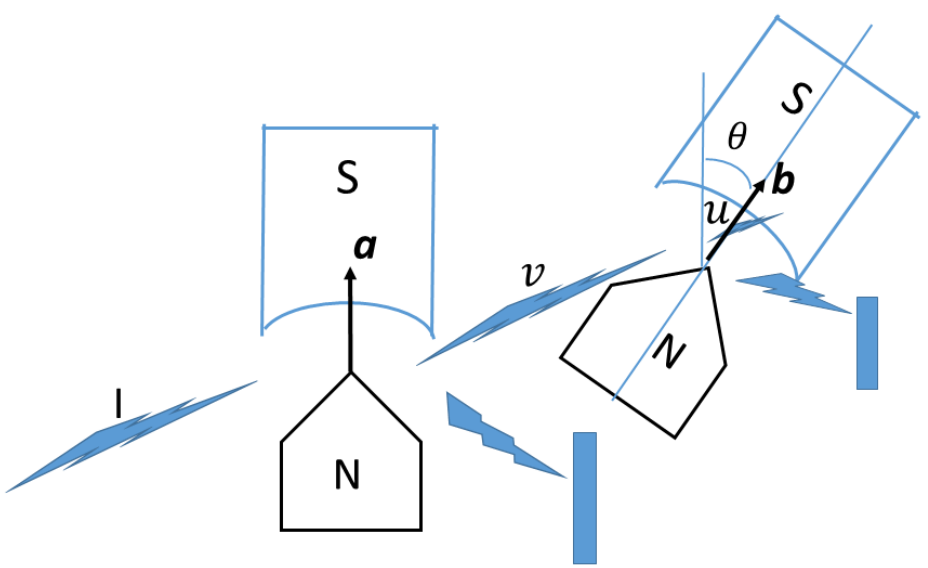

Figure 1. Setup of the Stern-Gerlach apparatuses to measure the transition probability between two states. The incoming beam $\mathrm{I}$ is in the direction perpendicular to both $\mathbf{a}$ and $\mathbf{b}$. It is split by the first SG apparatus into spin states $|a+\rangle=\varrho_{1}$ and $|a-\rangle=\bar{\varrho}_{1}$. The state $\varrho_{1}$ is measured by the second SG apparatus, rotated by an angle $\theta$ with respect to $\mathbf{a}$. We denote by $\varrho_{2}$ the state of particles going in the $\mid b+>$ direction after this measurement. The transition probability $\left[\varrho_{2} \mid \varrho_{1}\right]$ is the proportion of particles in state $\varrho_{1}$ that passes the second SG apparatus to state $\varrho_{2}$.

In Figure 2, we represent the minimal tripotents $v_{1}, v_{2}$ and their conjugates by using the decomposition (7) and (8).

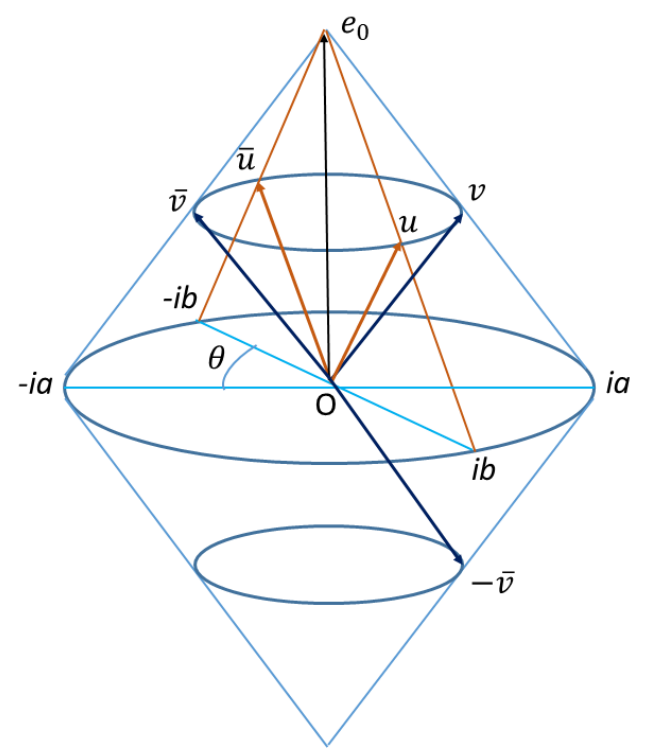

Figure 2. Decomposition (7) of minimal tripotents $v_{1}=\frac{1}{2}\left(e_{0}+i a\right)$ and $v_{2}=\frac{1}{2}\left(e_{0}+i b\right)$, for states with directions $\mathbf{a}$ and $\mathbf{b}$, respectively, with an angle $\theta$ between them. 
The transition probability between two pure states $\varrho_{1}$ and $\varrho_{2}$, denoted by $\left[\varrho_{2} \mid \varrho_{1}\right]$, is

$$
\left[\varrho_{2} \mid \varrho_{1}\right]=\hat{\varrho}_{2}\left(\varrho_{1}\right)=\frac{1}{2}\left\langle\varrho_{2} \mid \varrho_{1}\right\rangle,
$$

which coincides with the usual definition of transition probability in the operator formulation of quantum mechanics. For our example, using (8), the transition probability between $\varrho_{1}$ and $\varrho_{2}$ is

$$
\left[\varrho_{2} \mid \varrho_{1}\right]=\frac{1}{2}\left\langle\varrho_{2} \mid \varrho_{1}\right\rangle=\frac{1}{2}(1+\cos \theta)=\cos ^{2} \frac{\theta}{2}
$$

which coincides with the known formula for spin transition probability in quantum mechanics.

The spin state $\bar{\varrho}_{2}$ corresponds to the tripotent $\bar{v}_{2}$ with the spin direction direction $-\mathbf{b}$ opposite to the direction of $\varrho_{2}$, The meaning of this state is that the rotation of the particle is in the same plane as $\varrho_{2}$, but in the opposite direction. Since $\bar{\varrho}_{2}=e_{0}-i b$, the transition probability between $\varrho_{1}$ and $\bar{\varrho}_{2}$ is

$$
\left[\bar{\varrho}_{2} \mid \varrho_{1}\right]=\frac{1}{2}\left\langle\bar{\varrho}_{2} \mid \varrho_{1}\right\rangle=\frac{1}{2}(1-\cos \theta)=\sin ^{2} \frac{\theta}{2} .
$$

This shows that the sum of the transition probabilities from $\varrho_{1}$ to $\varrho_{2}$ and $\bar{Q}_{2}$ is one, as it should be.

\section{The Physical Meaning of the Non-Relativistic Spin}

Since pure states are non-decomposable, it is natural that the operator associated with a pure state is a minimal (rank one) tripotent, like $v=\hat{\varrho}$. This implies that the pure spin state $\varrho$ is as in (6). In non-relativistic quantum mechanics, we assume that the velocity of the measured particles is small with respect to the speed of light. For such particles, we may assume that its four-velocity is $e_{0}=(1,0,0,0)$. This is also the four-velocity of any particle in the comoving frame. Thus, the real part of a pure state (6) of a particle is its dimensionless four-velocity.

The imaginary part is a unit-length space-like vector representing its angular rotation. If we denote by $\omega$ the angular velocity of the rotating electron, then the Bloch vector of this spin state is $\mathbf{a}=\boldsymbol{\omega} / \omega$, and the imaginary part of the spin state $\varrho$ is $w=(0, \boldsymbol{\omega} / \omega)$. If we assume the validity of a Planck-type formula $E=\hbar \omega=m c^{2}$ also for an electron, then

$$
\omega=\frac{m c^{2}}{\hbar} .
$$

This follows also from the Schrodinger equation as follows. In the frame rotating together with the electron, its state is stationary and time independent. In the lab frame, the state is $\psi(t, \mathbf{x})=e^{i \omega t} \psi_{0}(\mathbf{x})$. Schrodinger's equation implies that $\omega=E / \hbar$. The imaginary part of the spin state $\varrho$ is

$$
w=\left(0, \frac{\hbar}{m c^{2}} \omega\right)
$$

We can express the properties of a pure spin state $\varrho$ as in (6) by use of the Minkowski metric, as follows:

$$
(\operatorname{Re} \varrho)^{2}=1,(\operatorname{Im} \varrho)^{2}=-1,<\operatorname{Re} \varrho \mid \operatorname{Im} \varrho>=0
$$

Note that when the directions of rotations of two pure states $v_{1}$ and $v_{2}$ differ by an angle $\theta$, the transition probability between $v_{1}$ and $v_{2}$ depends on $\theta / 2$. This is due to the fact that the spin state depends also on the four-velocity, and the states have a common four-velocity. See, for example, the states $v_{1}$ and $\bar{v}_{1}$ in Figure 2 which represent two electrons rotating in opposite directions about the common axis. The corresponding angular velocities differ by $180^{\circ}$, while the angle between $v_{1}$ and $\bar{v}_{1}$ is $90^{\circ}$. This is due to the common real part $e_{0}$. 
The vector $e_{0}$ represents a state of zero spin and can be decomposed as $e_{0}=v_{1}+\bar{v}_{1}=$ $v_{2}+\bar{v}_{2}$, a sum of two electrons with opposite spin. Thus, this vector may represent the state of a singlet [15] consisting a pair of electrons with opposite spin. Singlets were used to confirm the numerical correctness of a prediction of quantum mechanics, which violate Bell's inequality based on Einstein's hidden variable model. The prediction was the correlation $C(\theta)$ between the spin states measurements in directions $\mathbf{a}$ and $\mathbf{b}$ with an angle $\theta$ between them. The measurement was performed on two electrons $A$ and $B$ which formed a singlet and were separated without changing their spin. The electron A was measured by the first SG apparatus in direction a, and we denote its state after the measurement by $\varrho_{1}^{A}$ or $\bar{Q}_{1}^{A}$, depending on the observed result. Similarly, the electron B was measured by the second SG apparatus in direction $\mathbf{b}$, and we denote its state after the measurement by $\varrho_{2}^{B}$ or $\bar{Q}_{2}^{B}$, depending on the observed result.

The correlation $C(\theta)=P_{1}-P_{2}$, where $P_{1}$ is the probability that the two measurements gave the same result (either both were plus or both were minus), and $P_{2}$ is the probability that the two measurements gave the opposite results (one plus and the other minus). If the measurement of $\mathrm{A}$ gave the result plus, its spin state in the a direction is $\varrho_{1}^{A}$. However, since $\mathrm{B}$ was the companion of $\mathrm{A}$ in a singlet, its a component in the a direction is $\bar{Q}_{1}^{B}$. This was verified experimentally. The probability that the second measurement will be plus is the transition probability $\left[\varrho_{2}^{B} \mid \bar{\varrho}_{1}^{B}\right]$, which by (11) is $\sin ^{2} \frac{\theta}{2}$. Similarly, if the measurement of $\mathrm{A}$ gave the result minus, its spin state in the a direction is $\bar{Q}_{1}^{A}$, implying for $\mathrm{B}$ the state $\varrho_{1}^{B}$, and the probability that the second measurement will be minus is the transition probability $\left[\bar{\varrho}_{2}^{B} \mid \varrho_{1}^{B}\right]$, which by (11) is also $\sin ^{2} \frac{\theta}{2}$. Thus, $P_{1}=\sin ^{2} \frac{\theta}{2}$. The same argument for $P_{2}$, using (10), gives $P_{1}=\cos ^{2} \frac{\theta}{2}$ and

$$
C(\theta)=\sin ^{2} \frac{\theta}{2}-\cos ^{2} \frac{\theta}{2}=-\cos \theta
$$

which agrees with the well-known formula. This formula is used in the explanation of experiments testing the validity of quantum mechanics predictions by use of Bell's inequality, see ([15] pp. 586, 590).

\section{Physical Meaning of the Relativistic Spin State}

Consider now the spin of a particle moving with relativistic velocity with respect to a reference frame $K$. Note that in the Stern-Gerlach experiment and the anomalous Zeeman effect, the electron is a part of an atom and its velocity with respect to the lab frame is close to the speed of light. Thus, in order to understand these experiments, we need a relativistic description of the spin.

In the inertial frame $K^{\prime}$ co-moving with the particle, the pure spin state is given by (7) as $\varrho^{\prime}=e_{0}^{\prime}+i a^{\prime}$, which is an element of the complexified Minkowski space-time $M_{c}$. To define this state in $K$, we use the linear extension (as above) of the spacetime Lorentz transformation $\Lambda$ between $K^{\prime}$ and $K$ from Minkowski space-time $M$ to its complexification $M_{\mathcal{C}}$. Thus, the spin state of our moving particle in the frame $K$ is

$$
\varrho=\Lambda\left(\varrho^{\prime}\right)=\Lambda e_{0}^{\prime}+i \Lambda a^{\prime}=U+i S
$$

where (from the definition of $\Lambda$ ) $U$ is the four-velocity of our moving particle, and $S$ is the $4 \mathrm{D}$ spin vector in $K$, as defined in [6], which is also the Pauli-Lubansky spin vector. Note that the relativistic spin state $\varrho$ in our representation is a complex $4 \mathrm{D}$ vector and not a bi-spinor, as in the Dirac formulation. Moreover, we can consider the spin state as the total velocity of a rotating object, where the real part is the four-velocity and the imaginary part is its dimensionless angular rotation velocity. The spin direction $\mathbf{a}^{\prime}$ can be obtained by taking the spatial part of $\Lambda^{-1}(S)$. 
Since Lorentz transformations preserve the Minkowski dot product and norm, the state $\varrho$ will also satisfy the properties (14), that is,

$$
U^{2}=1, \quad S^{2}=-1,<U \mid S>=0 .
$$

Note any state of the form $U+i S$ with $U, S$ satisfying (17), describes a pure state of a particle moving with four-velocity $U$ and spin a defined by applying to $S$ the inverse Lorentz transformation defined by $U$. Thus, the set of all pure spin states forms a real five-dimensional manifold inside $M_{c}$.

In the relativistic limit, our model agrees with the following known result. Consider the spin state of a spin- $1 / 2$ particle moving with velocity close to the speed of light in a lab frame. Choose the $x$-axis as the direction of the velocity. Then $\Lambda$ is the matrix of a boost in the $x$-direction. Denote the spin of the particle in the comoving frame by $a=(0, a, b, d)$. Then, by (16), the spin $S$ in the lab frame is $S=(\gamma a v / c, \gamma a, b, d)$. If we assume that $a \neq 0$, then $S \sim(v / c, 1, b / \gamma a, d / \gamma a)$. In the relativistic limit $v \rightarrow c$, we have $\gamma \rightarrow \infty$ and $S \rightarrow(1,1,0,0)$ which is an eigenstate of "helicity," in agreement with a well-known result.

Using the Lorentz invariance of the dot product, the fact that spin measurements do change the velocity of the measured particle, and the definition of the transition probability, it follows that the transition probability between two relativistic pure spin states $\varrho_{1}$ and $\varrho_{2}$ of particles moving with the same velocity are defined by use of Minkowski dot product as

$$
\left[\varrho_{2} \mid \varrho_{1}\right]=\frac{1}{2}<\varrho_{2} \mid \varrho_{1}>.
$$

Since the Lorentz transformations preserve the Minkowski dot product, the transition probability of two pure spin states of particles moving with the same four-velocity $U$ will be the same as it is in the co-moving frame, implying that the transition probability is Lorentz invariant and is the same for any inertial observers.

The spin state operator of an electron describes both its angular and magnetic momentum. The angular momentum is connected with its mass $m$, while the magnetic moment is connected to its charge $q$. We define the state of the momentum as $P=m c \varrho=m c U+i m c S$. The real part $m c U$ coincides with the usual four-momentum of a moving particle in special relativity. The imaginary part $m c S$ represents the momentum of angular rotation, which, using (13), in the frame co-moving with the particle is $m c S=\left(0, \frac{\hbar}{c} \boldsymbol{\omega}\right)=(0, \mathbf{s})$, with $\boldsymbol{\omega}$ the angular velocity of the rotating electron and the angular momentum $\mathbf{s}$, as used in (1).

A moving and rotating charge $q$ generates an electromagnetic field. The $4 \mathrm{D}$ current is $q$ times the four-velocity. We define the electromagnetic momentum as $E=q \varrho=q U+i q S$. Its real part $q U$ is the current of the moving charged particle, while the imaginary part $q S$ represents the magnetic momentum generated by the rotation of the charged particle. In the co-moving frame, this magnetic momentum is $(0, \mu)$, with $\mu$ as in (1). Comparing the expression for the spatial part of $S$ in the co-moving frame from the angular momentum $S=\mathbf{s} / m c$ with the one from the magnetic momentum $S=\mu / q$, we obtain $\mu=\frac{q}{m c} \mathbf{s}$, which from (1) correspond to Landé factor $g=2$.

To define the evolution equation of the spin under an electromagnetic field described by an electromagnetic tensor $F$, we proceed as follows. The action of the field on the four-velocity $U$ is defined by the evolution equation

$$
\frac{d U^{\mu}}{d \tau}=\frac{q}{c m} F_{v}^{\mu} U^{v}
$$

and the action on the $4 \mathrm{D}$ spin vector $S$ is described by the BMT equation, which for $g=2$ is

$$
\frac{d S^{\mu}}{d \tau}=\frac{q}{c m} F_{v}^{\mu} S^{v}
$$


(see [6], Section 11.11). From the fact that a pure spin state $\varrho=U+i S$ evolves into a pure spin state, the evolution of the spin will satisfy the equation

$$
\frac{d \varrho^{\mu}}{d \tau}=\frac{q}{m c} F_{v}^{\mu} \varrho^{\nu} .
$$

It is shown in [6] Sections 11.8 and 11.11 that this equation and $g=2$ lead to the correct anomalous Zeeman effect and the fine structure splitting

Two particles can generate a mixed state only if they have the same four-velocity $U$ but may have different $4 \mathrm{D}$ spin vectors $S$. This implies that all pure and mixed spin states are $\varrho=U+i S$, with

$$
U^{2}=1,-1 \leq S^{2} \leq 0, \quad<U \mid S>=0 .
$$

It is obvious that any mixed state can be written as a convex combination of pure states.

Note that pure states are also stationary states, following from the fact that repeating the same measurement on the pure state will lead to the same outcome. If the particle is not affected by a force, it will preserve its four-velocity, linear momentum and current. Moreover, it will also preserve its angular velocity, angular momentum and magnetic moment.

\section{Summary}

Motivated by the algebraic formulation of quantum mechanics, we introduced a relativistic two-state space, which is a complexified Minkowski space $M_{c}$. We showed that it can serve as a state space for a two-state quantum system. The pure states and orthogonality can be described explicitly. These notions follow from a ternary product structure that we define on $M_{c}$. We can define transition probabilities between pure states, The Lorentz group acts on this space by linear extension of its action on the four-vectors of Minkowski space. The model is a relativistic model, and the states are complex vectors transforming as four-vectors.

We applied this model to represent the state of a spin $1 / 2$ particle. We have shown that the pure state of such a particle is of the form $\varrho=U+i S$, where $U$ is the four-velocity of the particle and $S$ is its $4 \mathrm{D}$ spin vector satisfying (17). This form of the pure spin vector follows from the indecomposability of the state, expressed by the minimality of the tripotent of the triple product.

We defined the notion of transition probability between two pure states and showed that the transition probability between two pure states moving with the same velocity is Lorentz invariant. Moreover, this transition probability coincides with the one predicted by quantum mechanics. We have shown that by use of our model, the correlation prediction used in tests of the validity of the quantum model by use of the Bell's Inequality coincide with the quantum one. The evolution equation of the spin state in an electromagnetic field is defined by (21), which correctly predicts the anomalous Zeeman effect and the fine structure splitting. We have shown that the Landé $g$ factor for such particles must be $g=2$.

We observed that in order for a spin state to be indecomposable, the momentum of angular rotation must have the same magnitude as the four-momentum. From (12), this angular frequency for an electron is $\omega \approx 10^{21} \mathrm{~s}^{-1}$.

What could explain such a phenomenon? In [16], it was shown that a field propagating with the speed of light generated by a single source has a pre-potential which is a complexvalued function on spacetime. For the electromagnetic field of a moving charged particle, the real part of the pre-potential's second derivative is the usual electromagnetic field generated by this source. In addition, however, the field is self-dual with respect to Hodge duality, or is of definite helicity. This implies that for such a field, there should be a connection between the electric and magnetic components. A particle can be identified with its electromagnetic field. Thus, we may expect a similar connection for the two parts of the spin state of the particle. 
Funding: This research received no external funding.

Acknowledgments: The author wants to thank Tzvi Scarr, Eric David Kramer, Eli Engelberg and Lawrence Horwitz for helpful discussions. He also wants to thank the referees for constructive and stimulating criticism, which helped to improve the paper.

Conflicts of Interest: The author declares no conflict of interest.

\section{References}

1. Cohen-Tannoudji, C.; Diu, B.; Laloë, F. Quantum Mechanics; John Wiley and Sons: Hoboken, NJ, USA, 2006; Volume 2, ISBN 978-0471-56952-7.

2. Gerlach, W.; Stern, O. Der experimentelle Nachweis der Richtungsquantelung im Magnetfeld. Z. Phys. 1922, 9, 349-352. [CrossRef]

3. Dirac, P.A.M. Principles of Quantum Mechanics, 4th ed.; International Series of Monographs on Physics; Oxford University Press: Oxford, UK, 1982; p. 255.

4. Pauli, W. The Connection Between Spin and Statistics. Phys. Rev. 1940, 58, 716-722. [CrossRef]

5. Horwitz, L.P.; Arshansky, R. On relativistic quantum theory for particles with spin 1/2. J. Phys. A Math. Gen. 1982, 15, L659. [CrossRef]

6. Jackson, J.D. Classical Electrodynamics, 3rd ed.; John Wiley \& Sons: Hoboken, NJ, USA,1998; ISBN 978-0-471-30932-1.

7. Thomas, L.H. The kinematics of an electron with an axis. Philos. Mag. 1927, 7, 1-23. [CrossRef]

8. Friedman, Y.; Russo, B. Geometry of the dual ball of the spin factor. Proc. Lond. Math. Soc. 1990, 65, 147-174. [CrossRef]

9. Friedman, Y.; Peralta, A.M. Representation of symmetry transformations on the sets of tripotents of spin and Cartan factors. arXiv 2021, arXiv:2101.00670.

10. Cartan, E. Sur les domains bornes homogenes de l'espace de n variables complexes. Abh. Math. Sem. Univ. Hamburg 1935, 11, 116-162. [CrossRef]

11. Friedman, Y. Physical Applications of Homogeneous Balls; Progress in Mathematical Physics 40; Birkhauser Boston, Inc.: Boston, MA, USA, 2005.

12. Whittaker, E.T. History of the Theories of Ether and Electricity; Longmans, Green: London, UK, 1910.

13. Friedman, Y.; Semon, M. Relativistic acceleration of charged particles in uniform and mutually perpendicular electric and magnetic fields as viewed in the laboratory frame. Phys. Rev. E 2005, 72 , 026603. [CrossRef] [PubMed]

14. Berestetskii, V.B.; Lifshitz, E.M.; Pitaevskii, L.P. Quantum Electrodynamics, 2nd ed.; Butterworth-Heinemann: Oxford, UK, 1982; Volume 4.

15. Ballentine, L.E. Quantum Mechanics: A modern Development, 2nd ed.; World Scientific: Singapore, 2015.

16. Friedman, Y.; Gootvilig, D.H.; Scarr, T. The Pre-Potential of a Field Propagating with the Speed of Light and Its Dual Symmetry. Symmetry 2019, 11, 1430. [CrossRef] 